\title{
Origine et diversité des ignames Dioscorea rotundata Poir
}

\author{
Comment le savoir-faire des paysans africains \\ leur permet d'utiliser la biodiversité sauvage dans l'agriculture
}

Roland Dumont ${ }^{1}$

Jeanne Zoundjihekpon ${ }^{2}$

Philippe Vernier ${ }^{1}$

1 Cirad

TA $180 / 04$

Avenue Agropolis

34398 Montpellier cedex

France

$<$ roland.dumont6@wanadoo.fr>

<philippe.vernier@cirad.fr>

2 Laboratoire d'écologie appliquée

Faculté des sciences agronomiques

Université d'Abomey-Calavi

06 BP 2083

Cotonou

Bénin

<jzoundjihekpon@pharaon-telecom.net>

\begin{abstract}
Résumé
La production mondiale des ignames est très majoritairement basée sur l'espèce Dioscorea rotundata, dont l'Afrique de l'Ouest est l'aire de culture principale. Cette espèce se divise en trois ensembles agronomiques et génétiques, chacun d'eux composé de nombreux cultivars polyclonaux. Les $D$. rotundata précoces, sexuellement fonctionnelles, sont issues de processus de domestication qui se poursuivent, aujourd'hui encore, sur diverses ignames existant à l'état sauvage : les espèces $D$. praehensilis et $D$. abyssinica, les hybrides entre ces espèces et $D$. rotundata, les hybrides intercultivars. Dans ce matériel végétal, le paysan capte épisodiquement des clones sauvages phénotypiquement proches de ses cultivars pour les incorporer à ceux-ci. Cette pratique accroît la diversité génétique des $D$. rotundata reproduites et multipliées par voie végétative, les dotant ainsi d'une capacité évolutive. L'origine des cultivars privés de sexualité reste incertaine, on se limite donc à évoquer quelques hypothèses.
\end{abstract}

Mots clés : Afrique ; biodiversité ; Dioscorea rotundata ; domestication ; igname.

Thèmes : amélioration génétique ; productions végétales ; ressources naturelles et environnement.

\begin{abstract}
Origin and diversity of Dioscorea rotundata Poir yams. How African peasants' knowledge makes it possible for them to use wild biodiversity in farming

The African species Dioscorea rotundata provides most of the world yam output which comes essentially from West Africa. This species is divided into three agronomic and genetic groups. Each of them comprises a large number of polyclonal cultivars. The early maturing $D$. rotundata cultivars are sexually functional. They come from on-going domestication of different species growing in the wild: D. praehensilis and D. abyssinica species, hybrids between both species and D. rotundata, intercultivar hybrids. Within this genetic material, farmers occasionally find wild phenotypes similar to their cultivated yams and mix them with their own material. This practise increases the genetic diversity of vegetatively propagated $D$. rotundata and introduces an evolving dimension. Regarding sexually non-functioning cultivars, their origins are still uncertain and we will limit our discussion to a few hypotheses.
\end{abstract}

Key words: Africa; biodiversity; Dioscorea rotundata; domestication; ignames.

Subjects: genetic improvements; natural resources and environment; vegetal productions.
Pour citer cet article : Dumont R, Zoundjihekpon J, Vernier Ph. Origine et diversité des ignames Dioscorea rotundata Poir. Comment le savoir-faire des paysans africains leur permet d'utiliser la biodiversité sauvage dans l'agriculture. Cah Agric 2010; 19: 255-61. DOI: 10.1684/ agr.2010.0411. 
elon la $\mathrm{FAO}^{1}$, la production mondiale d'ignames, évaluée en 2008, dépasserait 51,7 millions de tonnes, et l'Afrique occidentale assurerait $93 \%$ de ce total en ayant triplé sa production au cours des quatre dernières décennies. On estime que l'espèce Dioscorea rotundata Poir. fournit près de $90 \%$ de la production des ignames africaines. Malgré leur rôle économique majeur, ces ignames ont longtemps été négligées par la recherche scientifique. Toutefois, depuis un demi-siècle, de nombreuses études ont été réalisées; beaucoup ont porté sur l'agronomie, mais d'autres concernent la botanique, la domestication des ignames sauvages et le recueil des savoirs paysans. Plus récemment, des outils très performants ont permis à la génétique d'élucider la nature des relations entre ignames "sauvages " et cultivars $D$. rotundata. À la lumière des diverses informations concernant la diversité et l'origine de ces cultivars, nous tenterons de faire la synthèse des connaissances sur la domestication des ignames et de formuler des hypothèses susceptibles d'orienter la réflexion vers les zones d'ombre subsistantes.

\section{Domestication}

\section{des ignames sauvages en Afrique}

Le résultat essentiel de la domestication est l'espèce $D$. rotundata dont la dimension économique majeure a été soulignée. D'autres ignames cultivées ont été obtenues, en Afrique, à partir des espèces sauvages. Les plus communes sont $D$. bulbifera, $D$. dumetorum et D. cayenensis. Elles sont largement distribuées géographiquement et peuvent avoir une importance économique notable localement; c'est le cas pour les deux premières au Cameroun occidental et, curieusement, pour la troisième, dans l'île antillaise de La Jamaïque (Degras, 1986) où elle a été introduite par la traite esclavagiste.

La domestication des ignames est fondamentalement motivée par le besoin de substituer la culture à la cueillette pour

${ }^{1} \mathrm{FAO}, 2009$. http://faostat.fao.org [consulté le 28/12/2010]. assurer la sécurité alimentaire des familles. La productivité est un objectif majeur visé par les paysans, mais d'autres critères sont aussi importants: précocité de production, aptitude à la conservation, adéquation à la demande commerciale et, plus récemment, adaptation du matériel végétal à la culture continue des terres imposée par une pression démographique croissante et l'adoption de la culture attelée.

Cependant, la préoccupation essentielle est la qualité organoleptique des tubercules dont l'absence d'amertume constitue le critère principal. La taille des tubercules visée par les producteurs et les consommateurs constitue aussi un critère important L'utilisation alimentaire classique privilégie les tubercules volumineux $(\geq 1,5 \mathrm{~kg})$ qui conviennent particulièrement à la fabrication du foutou ou igname pilée, qui est la cuisine "noble" des ignames. Depuis moins d'un siècle, des productions régionales se sont tournées vers les cultivars produisant de petits tubercules convenant bien à la fabrication des cossettes séchées qui fournissent, après réduction en farine et cuisson rapide, une pâte souple et colorée, appelée " amala ". Au Nigeria et au Bénin, l'urbanisation a généré une forte demande commerciale pour ce produit (Bricas et al., 1997).

\section{Origine des ignames Dioscorea rotundata}

Il a d'abord été supposé que $D$. rotundata dérivait des espèces sauvages $D$. praehensilis et $D$. abyssinica, l'une forestière et l'autre savanicole (Miège, 1952 ; Coursey, 1967). L'idée est maintenant confirmée, pour l'Afrique de l'Ouest, par plusieurs travaux utilisant des marqueurs enzymatiques et moléculaires (Hamon, 1988; Terauchi et al., 1992; Ramser et al., 1997 ; Dansi et al., 2000, Scarcelli et al., 2006a; Scarcelli et al., 2006b; Chaïr et al., 2005; Tostain et al., 2005; Mignouna et al., 2002 ; Mignouna et al., 2003a, Mignouna et al., 2005).

Les parents sauvages des D. rotundata apparaissent génétiquement distincts avec, toutefois, le même nombre de chromosomes [2n = 40, $\mathrm{X}=20$ ] (Hamon et al., 1992) et un même haplotype (Chaïr et al., 2005; Tostain et al., 2005). Il s'agirait de deux taxons divergents d'un même ensemble génétique. En captant et en multipliant par voie végétative une partie de leur biodiversité, la domestication déterminerait une variation adaptative, pourvue de caractères phénotypiques particuliers, constituant un troisième taxon. Le tout pouvant être regardé comme un ensemble botanique composé de populations nanties, chacune, de caractéristiques phénotypiques et génétiques intrinsèques autour d'un compartiment intermédiaire regroupant des génotypes interféconds.

\section{Diversité des ignames Dioscorea rotundata}

D. rotundata est un matériel végétal créé pour l'agriculture. Les paysans d'Afrique occidentale y distinguent trois groupes sur la base de divers critères d'usage dont les principaux sont l'époque de récolte et l'aptitude à la conservation.

- Les cultivars précoces sont récoltés deux fois au cours du cycle cultural annuel. Une première production intervient 4 à 6 mois après la levée; elle compte un à deux tubercules volumineux et longs (30-100 cm) destinés à la consommation. Au cours de la période végétative restante (2 à 4 mois), une seconde tubérisation fournit un agrégat des petits tubercules utilisés pour la reproduction végétative. Trente-huit des 54 cultivars précoces florifères, relevés au Bénin par Dansi et al. (1999) sont femelles avec une floraison d'intensité variable mais toujours fertile. Dans le même pays, Tostain et al. (2007) identifient 79 cultivars précoces florifères, dont 40 mâles mais un fort déséquilibre entre les sexes apparait à l'échelle régionale : $71 \%$ des cultivars sont mâles dans le Nord-Ouest contre $27 \%$ dans le Nord-Est.

- Les cultivars tardifs sont toujours récoltés au terme du cycle végétatif. On distingue trois morphotypes. Le plus commun fournit deux à quatre tubercules volumineux, bien individualisés. Les deux autres morphotypes correspondent à des cultivars particuliers. Les ignames Allassora (Togo, Bénin) donnent quatre à six tubercules de faible diamètre. Chez les ignames Kokoro (Nigeria, Bénin), cultivées pour fabriquer des cossettes, la plante produit trois à huit tubercules courts et globuleux, soudés au niveau de leur tête; l'ensemble ressemblant curieusement à la production 
de seconde récolte obtenue chez les ignames précoces. Dans les deux derniers cas, la taille de l'appareil aérien diminue et la réponse à l'haptotropisme caulinaire, inductrice de volubilité, s'affaiblit ou disparaît; ce stade ultime s'observe chez le cultivar Tam Saan (type Allassora), "l'igname arachide " du pays Bariba béninois, dont la longueur des tiges reste inférieure à 1 mètre. La productivité régresse; un suivi sur cinq cultivars tardifs et autant de cultivars précoces, opéré au cours de trois campagnes successives (1995-1997) en paysannat nord-béninois, indique un rendement moyen de $14,4 \mathrm{t} /$ ha pour les premiers contre 23,9 t/ha pour les seconds (Vernier et Dossou, 2000). Chez les cultivars tardifs, le développement limité des tubercules apporte un avantage, car il permet une récolte étalée sur la saison sèche alors que le sol s'est durci. L'aptitude à la conservation se maintient alors sur 5 à 6 mois contre 1 à 2 mois pour les cultivars précoces. Une partie de la production est consommée, l'autre est utilisée comme semence. La floraison mâle semble générale chez les cultivars tardifs (Tostain et al., 2007) mais l'interfécondité avec les $D$. rotundata précoces disparaît. On ignore si cela est dû à une stérilité pollinique, à une incompatibilité génétique ou à une autre barrière de reproduction. Cette absence d'interfécondité a été vérifiée sur quatre cultivars tardifs (Dumont et al., 2005), mais on constate aussi que, malgré leur abondance dans l'agriculture du Nord Bénin (Dumont, 1997 ; Baco, 2000), les cultivars mâles n'y pollinisent pas les ignames sauvages femelles (Scarcelli et al., 2006b).

- Les cultivars intermédiaires sont exploitables précocement ou tardivement selon la qualité de l'environnement et la stratégie des paysans. Le nombre de tubercules varie de 1 à 3 avec une longueur réduite $(30 \mathrm{~cm})$, ce qui affecte le potentiel de rendement. En pays Sénoufo ivoirien, Stessens (2002) chiffre ainsi à 33\% la régression de rendement entre les cultivars Krenglé (intermédiaire) et Wacrou (précoce). L'aptitude à la conservation avoisine celle des cultivars tardifs. Nous avons observé des floraisons régulières, irrégulières, voire même exceptionnelles, avec des cas de parthénocarpie ou de stérilité florale. D'autres troubles sont plus rares : fasciation des jeunes tiges, production de bulbilles, apparition des caractéristiques foliaires de l'espèce sauvage D. sagittifolia (cas particulier du cultivar Boni Ouré nord-béninois), monoécie et inversion de la sexualité toujours orientée vers le sexe mâle (Dumont et al., 2005). Chez le cultivar Ahimon ${ }^{+}$, Dansi et al.(1999) ont obtenu une plante femelle et une autre mâle en plantant deux portions d'un même tubercule. Ces différentes observations font penser à un fonctionnement biologique sujet à l'instabilité sporadique.

Dès 1979, les caractères morphologiques avaient permis à Miège de distinguer imparfaitement les cultivars précoces des cultivars tardifs. Ce résultat est aujourd'hui avalisé par les marqueurs moléculaires. La structuration phylétique établie par Scarcelli (2005) reflète et hiérarchise un clivage génétique au sein des $D$. rotundata du Nord-Bénin où les cultivars intermédiaires s'intercalent génétiquement entre les deux autres groupes de cultivars. Mignouna et al. (2005), Tamirou et al. (2007), Tostain et al. (2007) confirment ce clivage génétique, et son indépendance par rapport au sexe est précisée par la dernière de ces études. On retiendra donc que les trois groupes de $D$. rotundata correspondent à des populations distinctes. Parmi les ignames précoces du Nord-Bénin, le dendrogramme établi par Tostain et al. (2007) montre une distribution en ensembles multicultivars individualisés suggérant des origines parentales différentes.

Le caractère polyclonal des cultivars a par ailleurs été révélé par plusieurs travaux (Hamon, 1988 ; Dansi et al., 2000 ; Mignouna et Dansi, 2003a ; Tostain et al., 2007). Chaque cultivar est composé de clones génétiquement voisins, certains étant hégémoniques et la diversité intracultivar varie peu dans un même terroir (Scarcelli, 2005). On ignore si cette situation se modifie avec l'élargissement de la distribution géographique ou le changement des conditions culturales.

\section{Domestication conduisant aux ignames $D$. rotundata}

Curieusement, les pratiques paysannes de domestication n'ont pas été remarquées par les botanistes et chercheurs de diverses disciplines agronomiques ou anthropologiques ayant travaillé en Afrique occidentale. Il a fallu attendre la fin du siècle dernier pour avoir un début d'information sur le sujet (Hamon, 1988 ; Chikwendu and Okezie, 1989) et c'est plus tard encore qu'une première étude approfondie lui a été consacrée (Dumont et Vernier, 1997 ; et Dumont et Vernier, 2000).
Au Bénin et au Nigeria, 40 à $90 \%$ des paysans interrogés savent comment obtenir des cultivars de $D$. rotundata en partant des espèces sauvages $D$. praehensilis et $D$. abyssinica. Cependant, la mise en pratique de ce savoir varie entre 1 et $20 \%$ des exploitations (Dumont et Vernier, 1997 ; Dumont et Vernier, 2000 ; Baco, 2000 ; Tostain et al., 2003 ; Vernier et al., 2003).

Deux enquêtes (Dumont et Vernier, 2000 ; Mignouna, Dansi et Mayong, 2003 b) précisent que la domestication maîtrisée par le paysan béninois est celle qui aboutit aux cultivars précoces, sexuellement fonctionnels. Les cultivars intermédiaires ou tardifs n'apparaissent guère dans les produits de domestication; le ou les processus les générant échapperai(en)t au paysan. On a donc deux situations qui doivent être examinées séparément.

\section{Domestication conduisant aux $D$. rotundata précoces}

Elle met en œuvre deux processus distincts dans leur fonctionnement et leur temporalité. L'un est l'échange réciproque de gènes entre des cultivars $D$. rotundata et certains compartiments des ignames sauvages; il s'exerce sur le long terme et génère des combinaisons génétiques exploitables par le second processus. Celui-ci, piloté par le paysan, intervient périodiquement à l'intérieur du premier; en quelques campagnes de culture, il transforme les caractères morphologiques des ignames sauvages sans recourir à la sexualité.

\section{Échanges géniques entre ignames sauvages et cultivars}

La migration gamétique a été démontrée entre ignames sauvages mâles et cultivars femelles (Tostain et al., 2007 ; Scarcelli et al., 2006a; Scarcelli et al., 2006b). Les derniers auteurs indiquent que 16 et $37 \%$ des domestications observées au Bénin présentent des caractères hybrides entre $D$. praehensilis ou $D$. abyssinica et D. rotundata.

Une migration zygotique va des champs cultivés vers le milieu sauvage via les graines ailées transportées par les vents, certaines venant de croisements intercultivars (Scarcelli et al., 2006a ; Tostain et al., 2007). Ceux-ci prouvent l'existence de cultivars mâles fertiles mais leurs gènes n'ont pas été décelés parmi les ignames sauvages (Scarcelli et al., 2006b). En fait, ces cultivars seraient des ignames précoces dont la floraison est déprimée par la 
concomitance de la première récolte. Des gamètes mâles rejoignent probablement la flore sauvage mais leur rareté réduit la probabilité de les détecter.

L'importance des migrations zygotiques annuelles est subordonnée à la surface consacrée aux cultivars sexuellement actifs. Deux enquêtes réalisées, l'une au Bénin septentrional (Dumont, 1977) et l'autre au nord de la Côte d'Ivoire (Stessens, 2002), montrent que chaque exploitation cultive 800 et 4500 individus produisant respectivement 4800 et 27000 graines. On calcule ainsi qu'un terroir fournirait plusieurs millions de graines, dont une partie intégrerait la population des ignames sauvages. Les hybrides présumés inter- $D$. rotundata, recueillis à l'état sauvage, fournissent d'ailleurs $47 \%$ des clones en cours de domestication au Nord-Bénin contre $20 \%$ dans le sud du pays (Scarcelli et al., 2006a). L'écart dépend, probablement, du degré d'introgression entre ignames sauvages et cultivars. Dans le nord, on se trouve dans une civilisation agraire fondée sur l'igname dont l'ancienneté est attestée par une vaste dimension culturelle. Dans le sud, la domestication a été engagée plus récemment par la demande commerciale (Bricas et al., 1997).

Une pression démographique croissante conduit à la réduction ou à l'abandon de la jachère arborée. Cette évolution érode les effectifs des ignames sauvages et parfois entraîne leur quasi-disparition. Nous avons rencontré cette régression sévère chez diverses ethnies : Kabyé et Kotokoli du Togo, Yom et Nago du Bénin, Sénoufo du Burkina Faso et Yorouba du Nigeria. Cette situation semble favoriser l'obtention de cultivars nouveaux. Ainsi, parmi les 38 domestications béninoises de ces 30 dernières années, 24 sont l'œuvre des ethnies Yom et Nago (Vernier et Dansi, 2006) attachées à des environnements dégradés.

En tout état de cause, la répétition des flux géniques partant de l'agriculture conduit à l'anthropisation des ignames sauvages, son degré étant conditionné par l'abondance des cultivars $D$. rotundata sexuellement actifs et par l'ancienneté de leur culture.

\section{L'intervention du paysan et ses effets}

Les graines des ignames sauvages et cultivées ne sont pas exploitées par les paysans domesticateurs car elles fournissent des résultats très aléatoires (Dumont et al., 2005). Par des observations empiri- ques, les paysans ont établi qu'il est plus efficace d'utiliser les tubercules des ignames sauvages en procédant comme expliqué ci-dessous.

- Un tri est effectué parmi les populations naturelles de D. abyssinica ou de D. praebensilis en examinant l'appareil végétatif pour rechercher les marqueurs reconnus corrélés au succès de la domestication par l'expérience ancestrale : forme et coloration de la feuille, diamètre et coloration de la tige, type de spinescence. Ensuite, le choix des tubercules s'opère selon divers critères : forme générale, volume, absence de digitations, couleur de la peau, rareté des radicelles et absence d'amertume (Baco, 2000 ; Dumont et al., 2005).

- Les tubercules retenus sont mis en culture. Ce transfert entraîne l'ennoblissement de la plante, c'est-à-dire les transformations morphologiques profondes correspondant au processus de domestication. Le tubercule est raccourci, son diamètre s'accroît, sa forme devient plus régulière et la quantité des radicelles épidermiques diminue (Chikwendu et Okezie, 1989). Parallèlement, le développement caulinaire est réduit en affectant peu la surface foliaire globale. Selon les paysans, l'ensemble de ces transformations s'obtient en trois à cinq campagnes de culture, tout en restant réversible. Deux contraintes techniques sont nécessaires pour les générer et les maintenir (Dumont et Vernier, 1997 ; Dumont et Vernier, 2000 ; Baco, 2000 ; Tostain et al., 2003 ; Dumont et al., 2005).

L'une intervient à la plantation : un obstacle (fragment de poterie, de calebasse ou de tôle, pierre plate) est inséré sous le semenceau placé dans la butte. Cette pratique aurait un rôle sélectif ; la plante obtenue serait rebelle à l'ennoblissement quand son tubercule déborde l'obstacle ou s'enroule sur sa surface. Cette forme de sélection concerne surtout les D. abyssinica.

L'autre technique est la double récolte, exercée 3 à 5 mois après la levée et répétée au cours des deux ou trois cultures suivantes. L'opération permet l'ennoblissement des $D$. praehensilis et compléterait l'effet de l'obstacle chez les D. abyssinica.

- Les clones ennoblis sont évalués en conditions d'agriculture réelle, pendant plusieurs années successives. Certains apparaissent comme des cultivars nouveaux (Dumont et Vernier, 1997 ; Dumont et Vernier, 2000 ; Baco, 2000 ; Scarcelli, 2005). Pourtant, peu d'entre eux connaissent une large diffusion. Les informations recueillies au Nord-Bénin ${ }^{2}$ indiquent une ancienneté supérieure au siècle pour les cultivars exploités actuellement, en exceptant, toutefois, Ourou Yensingué, Kokouma, Ahimon et quelques introductions d'origine connue. En fait, le domesticateur nord-béninois recherche surtout des clones phénotypiquement proches des cultivars déjà existants pour les cultiver avec ceux-ci (Dumont et Vernier, 1997 ; Dumont et Vernier, 2000 ; Baco, 2000). La même pratique existe dans le Sud-Ouest de l'Éthiopie (Hildebrand, 2003) et est confirmée au Bénin (Scarcelli et al., 2006b) où, selon Tostain et al. (2007), elle expliquerait la plus grande partie de la diversité intracultivar.

Des enquêtes conduites au Bénin (Dumont, 1997 ; Dumont et Vernier, 1997 ; Dumont et Vernier, 2000 ; Baco, 2000 ; Vernier et al., 2003) montrent que près de $80 \%$ de la production des D. rotundata précoces vient de cultivars déconnectés de la domestication, plus productifs parce que la régression ou la perte de sexualité avantage la tubérisation. Dans les bassins de production depuis longtemps riches en matériel végétal, la création de cultivars n'est pas un besoin majeur aussi longtemps que les conditions de culture restent stables. Le plus souvent, l'objectif de la domestication serait l'amélioration et/ou la pérennisation de cultivars déjà existants.

Les enquêtes (Dumont et Vernier, 1997 ; Dumont et Vernier, 2000) avaient d'ailleurs permis d'estimer que 1600 paysans Bariba introduisent épisodiquement des clones, issus de la flore sauvage, dans six cultivars communs sur un territoire de $10000 \mathrm{~km}^{2}$ et dont l'origine remonterait à plus de trois générations humaines, d'après les informations recueillies auprès de la population locale.

Ainsi regardée, la domestication des $D$. rotundata précoces apparaît comme une dynamique collective nécessitant une série d'allers-retours entre ignames sauvages et cultivars. À chaque étape, les choix s'exercent sur un compartiment de la population sauvage dont la valeur moyenne se trouve améliorée par des flux géniques venus d'une agriculture ayant abondamment multiplié les sélections précédentes par voie végétative. Cette situation fournit l'opportunité d'enrichir la diversité génétique des cultivars, celle-ci pouvant, par ailleurs, être

\footnotetext{
$\overline{2}$ Dumont, observation personnelle.
} 
occasionnellement élargie par des mutations (Scarcelli, 2005). Cette dernière idée semble illustrée par les cultivars Morokorou et Kokouma du Nord-Bénin. Ils diffèrent par la forme du tubercule tout en apparaissant génétiquement très proches dans le dendrogramme construit par Tostain et al., 2007. Les paysans disent que le premier vient des ignames sauvages mais hésitent sur l'origine du second, perçu comme une innovation dans les années $1960^{3}$.

En passant par la sexualité, la domestication aurait d'autres effets bénéfiques. D'abord, l'élimination des mutations délétères (Allano et Clamens, 2000) et des virus (Thouvenel et Fauquet, 1978). Ensuite, un réajustement de la valeur d'équilibre homéostatique intervenant en cas de modifications environnementales (Binder, 1972). Enfin, les paysans nous ont dit attendre des innovations avantageuses de la domestication et c'est, en fait, leur motivation consciente. Au Nord-Bénin, l'idée concerne surtout la parfaite aptitude à la fabrication du foutou, généralement présentée comme longue à obtenir. Dans notre enquête (Dumont et Vernier, 1997; Dumont et Vernier, 2000), le cultivar Ourou Yensingué (littéralement: qui fait des boules) vient en tête des ignames encore travaillées par la domestication ; la précocité de production a été améliorée, mais il reste à éliminer les indurations liégeuses affectant la qualité du foutou.

Parallèlement aux effets enrichissants de la domestication, il faut envisager l'hypothèse d'une sélection excluant les combinaisons génétiques désavantageuses. Au niveau du champ et de l'année, chaque cultivar précoce produit une proportion variable de plantes trop faibles pour être traitées en double récolte, ce qui les écarte de la reproduction végétative. La plupart de ces évictions s'expliqueraient par l'hétérogénéité physique et chimique du sol, la surcharge virale et le stress hydrique. Mais on peut aussi subodorer des causes génétiques comme l'effet dépressif d'une mutation altérant la croissance du tubercule ou encore l'apparition d'une limite dans la capacité d'adaptation au changement de l'environnement.

Le cultivar précoce aurait ainsi une dimension évolutive, déjà reconnue par Hamon (1988). Elle résulterait de l'intégration chronique d'innovations génétiques tirées de la sexualité des ignames

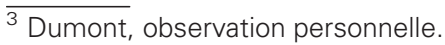

sauvages et d'une probable érosion génétique impulsée par les pratiques agricoles et/ou les changements environnementaux. Cette combinaison de pressions couvrant de nombreuses décennies, voire plusieurs siècles, permet des ajustements génétiques successifs. La diversité génétique du cultivar peut se modifier profondément dans le temps alors que son nom reste invariable.

\section{Origine des $D$. rotundata}

intermédiaires et tardives

L'itinéraire technique conduisant aux D. rotundata précoces devient impraticable puisque la déficience de sexualité raréfie ou supprime l'échange de gènes avec les ignames sauvages. L'origine des cultivars intermédiaires et tardifs reste spéculative. Néanmoins, quelques observations et résultats expérimentaux peuvent alimenter la réflexion sur le sujet.

- Les cultivars intermédiaires pourraient être des cultivars précoces en voie de sénescence. Les deux formes d'ignames restent proches sur plusieurs plans. D'abord, les phénotypes se séparent difficilement (Dansi et al., 1999). Ensuite, deux résultats de nature génétique vont dans le même sens. Scarcelli (2005) situe deux cultivars intermédiaires au voisinage d'un cultivar précoce à sexualité épisodique tout en les séparant de trois cultivars précoces fleurissant régulièrement. Tostain et al. (2007) ont aussi relevé une étroite proximité génétique entre cultivars précoces et cultivars intermédiaires. Enfin, sous réserve de fleurir au moins sporadiquement, la plupart des cultivars intermédiaires restent fertiles. Ainsi, en parcourant les monocultures de Krenglé ${ }^{+}$, fréquentes dans la savane ivoirienne, avons-nous souvent observé une fructification abondante prouvant leur compatibilité génétique avec l'offre gamétique environnementale. De plus, nous avons réalisé une série d'hybridations entre Krenglé et D. praehensilis ; la génération F1 a fourni des tubercules longs, munis de racines épineuses et un appareil végétatif vigoureux avec un feuillage d'un vert très sombre, étranger aux parents. Ce matériel végétal est conservé par la vitrothèque du Centre national de la recherche agronomique (CNRA) de Côte d'Ivoire.

- Les ignames tardives n'ont jamais été signalées à l'état sauvage et elles disparaissent, dans un délai de 2 à 4 ans, si on les introduit dans le milieu sauvage ${ }^{4}$.

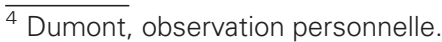

En Côte d'Ivoire où le démarrage des domestications serait historiquement récent puisqu'attribué à l'émigration Akan, partie du Ghana au XVII ${ }^{\mathrm{e}}$ ou XVIII $^{\mathrm{e}}$ siècle (Rougerie, 1982), les cultivars tardifs sont rares (Hamon, 1988). Cela expliquerait l'intérêt accordé à l'espèce D. alata (cas unique en Afrique occidentale) introduite dans un passé vieux d'environ cinq siècles pour Burkill mais beaucoup plus ancien pour Chevalier (In: Miège, 1952). Inversement, au Nigeria où des écrits portugais attestent du commerce des ignames dès 1491 (In : Coursey and Coursey, 1971) et donc une domestication encore plus ancienne, les cultivars tardifs sont nombreux, très diversifiés, productifs et toujours appropriés à la fabrication du foutou. La même richesse en cultivars existe au Togo et au Bénin selon les recensements opérés localement (Dansi et al., 1999).

Les réflexions précédentes conduisent à trois hypothèses concernant la genèse des cultivars tardifs avec des combinaisons possibles entre les facteurs évoqués.

\section{La suite d'une sénescence engagée chez les cultivars intermédiaires}

Dans un terroir Bariba, huit des 36 cultivars rencontrés occupent $60 \%$ de la surface consacrée aux ignames (Dumont, 1997). Dans un autre terroir, situé à environ $70 \mathrm{~km}$ du premier, 15 des 72 cultivars identifiés, couvrent $70 \%$ des surfaces cultivées en ignames (Baco et al., 2004). Dans les deux cas, le nombre de cultivars D. rotundata apparaît élevé mais une faible partie assure la majorité de la production. Le solde se subdivise de la façon suivante: d'une part, les cultivars en cours d'évaluation que sont les produits de domestication et les introductions récentes ; d'autre part, des cultivars très anciens protégés par la déontologie ancestrale. À titre d'exemples, la mémoire collective Bariba (Dumont et al., 2005) permet d'approcher l'âge des cultivars Gambari gnignou et Yon bouanri, marginaux dans l'agriculture actuelle. L'un aurait été apporté par le commerce caravanier ayant, selon Lombard (1965), traversé le Bénin septentrional jusqu'au début $\mathrm{du} \mathrm{XIX}^{\mathrm{e}}$ siècle pour relier le Nord du Nigeria au royaume du Ghana ; l'autre serait un cadeau offert à l'occasion d'un mariage aristocratique remontant à plus de huit générations.

La sénescence des ignames est une notion floue. Pour l'approcher, on se référera au cultivar Kourotokokouragourouko du pays Bariba. Selon Baco (2000), ce nom 
signifie " la femme âgée jamais vieille ". On peut en tirer deux informations. D'abord, le caractère féminin du nom l'associe aux cultivars tardifs considérés comme femelles par le paysan (Dumont et al., 2005). Ensuite, l'image réfléchie par le nom révèle le cas, probablement exceptionnel, d'un cultivar écarté de la normalité en restant productif malgré son grand âge. Autrement dit, la sénescence des cultivars est une réalité qui mène à leur marginalisation. Les paysans ramènent ce phénomène à une "fatigue ", mais on ignore sa nature et son évolution.

\section{Un décalage entre les époques de domestication}

Tostain et al. (2002) indiquent que les espèces sauvages $D$. praehensilis et D. abyssinica sont génétiquement structurées dans l'espace. À l'échelle du Bénin, Tostain et al. (2007) révèlent une régionalisation dans la distribution géographique des cultivars D. rotundata. Par ailleurs, Tamiru et al. (2007) montrent que les D. rotundata éthiopiennes sont génétiquement étrangères à leurs homologues ouest-africaines. Ces structurations dans l'espace se doubleraient de déphasages dans le temps. Selon Birnbaum (2007), la paléoclimatologie indique que dans le passé, l'Afrique occidentale a connu une végétation forestière dépassant la latitude $10^{\circ}$, suivie d'une inversion vers la savane arborée à partir de 3000 BP. En suivant cette dynamique régressive, encore active de nos jours, les domestications auraient utilisé une succession d'ignames sauvages génétiquement refaçonnées au cours de l'évolution générale. Des cultivars très anciens pourraient ainsi se démarquer d'autres plus récents et des ignames sauvages actuelles.

\section{Une accumulation de mutations}

Dès 1987, Hamon avait supposé que les mutations expliquaient la vaste diversité génétique observée chez certaines D. rotundata. De son côté, Scarcelli (2005), relève l'abondance des mutations présumées chez les cultivars plus ou moins privés de sexualité et on peut penser que l'effet de cette carence s'accroît avec le vieillissement du matériel végétal. Enfin, Tostain et al. (2007) indiquent un excès de génotypes hétérozygotes chez les cultivars mâles et tardifs, collectés dans l'Atacora (N-O Bénin): cette situation est attribuée aux conditions stressantes de l'agriculture locale.
Deux puissants facteurs de stress accompagnent la production des D. rotundata. D'abord, les tubercules subissent des chocs thermiques parce que les terres cultivées sont exposées à l'ensoleillement. Ensuite, le sectionnement annuel des tubercules pour la multiplication et la plantation à l'occasion de double récolte répète des mutilations physiologiquement stressantes.

L'accumulation des mutations, comme les deux hypothèses précédentes, ne s'applique probablement pas aux cultivars Allassora et Kokoro. Ceux-ci semblent être apparus spontanément dans l'agriculture ou son environnement immédiat car nous ne les avons jamais rencontrés en déterrant des milliers d'ignames sauvages $^{5}$. Leur distribution géographique, antérieure à l'apparition de la demande commerciale, est clairement liée à une forte pression foncière sans pouvoir affirmer son rôle déterminant. Ces cultivars ont pu être privilégiés localement parce que leur rusticité convient aux environnements dégradés. Au nordouest du Bénin, l'ethnie Bariba a récemment adopté des cultivars cultivés dans le pays Yom voisin, dans les conditions d'une agriculture semi-sédentarisée et des pratiques de culture attelée (Dumont, 1997).

Les cultivars intermédiaires et tardifs restent soumis à de fortes pressions de sélection. D'abord, le paysan peut tirer parti des mutations ou autres variations génétiques, sur la base de leur expression phénotypique et obtenir ainsi des variants ou des cultivars nouveaux. Ensuite, des pratiques traditionnelles sont mises en oeuvre, à la plantation, pour garantir un haut niveau de rendement. Dans de nombreux cas, les plantes les plus productives fournissent, de façon supplémentaire, un petit tubercule utilisé pour la reproduction. Toutefois, l'insuffisance fréquente de telles semences nécessite le recours à la fragmentation des tubercules de calibre moyen $(\geq 0,75 \mathrm{~kg})$, ayant une faible probabilité d'être issus de plantes peu productives. Ces pratiques permettraient d'éliminer chaque année les individus devenus peu performants. Ainsi, la multiplication végétative laisserait-elle la porte ouverte à des interventions permettant de maintenir, dans une certaine mesure, la potentialité évolutive des cultivars privés de sexualité.

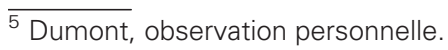

\section{Conclusion}

La plupart des espèces cultivées actuelles ont une origine masquée par un passé très ancien ; l'identité de leurs parents sauvages comme les mécanismes de domestication les ayant produites ne peuvent être approchés que par des voies indirectes, avec parfois des incertitudes et des controverses.

Avec la domestication des ignames sauvages conduisant à l'espèce Dioscorea rotundata, l'originalité de l'Afrique est d'offrir un cas contemporain et observable. Il révèle une haute technicité des agriculteurs africains dans la gestion de la biodiversité guidée par un savoir-faire resté insoupçonné jusqu'à un passé récent. À notre connaissance, la recherche scientifique n'a pas rencontré d'autre cas semblable jusqu'à présent.

Parce qu'elles sont encore utilisées de nos jours sur un large espace géographique, ces pratiques de domestication conduisant aux $D$. rotundata précoces restent accessibles aux études. Elles constituent un modèle concret permettant de comprendre comment on passe des plantes sauvages aux plantes cultivées à multiplication végétative. De plus, cette situation, génératrice d'hypothèses, fournit la possibilité rare de confronter les outils de la génétique moderne, notamment les analyses moléculaires, à un savoirfaire empirique ancestral. Cette perspective offre un champ d'investigations susceptible de fédérer un large éventail de disciplines scientifiques allant de la biologie aux sciences sociales.

Parallèlement, il deviendrait envisageable d'élargir la portée géographique des études, jusqu'ici centrées sur le Bénin. Trois directions nouvelles présenteraient, chacune, un intérêt particulier. L'Afrique centrale ouvre l'accès à un matériel sauvage et cultivé moins anthropisé. L'Éthiopie et l'Afrique occidentale permettent de comparer deux processus de domestication, a priori semblables mais fortement dissociés dans l'espace. Enfin, en ayant accueilli les $D$. rotundata africaines depuis plusieurs siècles, l'Amérique latine et les Caraibes offrent l'opportunité d'étudier leur évolution génétique en dehors de leur écosystème d'origine.

\section{Références}

Allano L, Clamens A. L'évolution. Des faits aux mécanismes. Collection Sciences de la Vie et de la terre. Paris : éditions Ellipses, 2000. 
Baco MN. La domestication des ignames sauvages dans la Sous-Préfecture de Sinende: savoirs locaux et pratiques endogènes d'amélioration génétique des Dioscorea abyssinica Hochst. Thèse d'ingénieur agronome, université nationale du Bénin, faculté des sciences agronomiques, Cotonou, Bénin, 2000.

Binder E. La génétique des populations. Collection Que sais-je? n॰ 1 283. Paris: Presses universitaires de France, 1972.

Birnbaum Ph. Mali, un oasis pour la biodiversité. 2007. www.futura-sciences.com/fr/comprendre/ dossiers/doc/développement-durable/d/mali

Bricas $N$, Vernier $\mathrm{P}$, Ategbo $\mathrm{E}$, et al. Le développement de la filière cossettes d'igname en Afrique de I'Ouest. Cahiers de la Recherche Développement $1997 ;(44)$ : 100-14.

Chaïr H, Agbangla C, Marchand JL, Daïnou O, Noyer JL. Use of cpSSRs for the characterisation of yam phylogeny in Benin. Genome 2005: 674-84.

Chikwendu VE, Okezie CEA. Factors responsible for the ennoblement of african yams : inferences from experiments in yam domestication. Foraging and farming: the evolution of plant exploitation. World archeological congress, Southampton, England, September 1986. Harris DR, Hillman GC, eds. London: Academic division of Unwin Hyman Ltd, 1989.

Coursey DG. Yams. An account of the Nature, Origins, Cultivation and Utilisation of the Useful Members of the Dioscoreaceae. Tropical Agricultural Series. London : Longmans; Green and Co. Ltd, 1967.

Coursey DG, Coursey CK. The New Yams festivals of West Africa. Anthropos 1971 : 444-84.

Dansi A, Mignouna HD, Zoundjihekpon J, Sangare A, Asiedu R, Quin FM. Morphological diversity, cultivars groups and possible descent in the cultivated yams Dioscorea cayenensis- $D$. rotundata complex in Benin Republic. Gen Res Crop Evol 1999; 46 : 371-88.

Dansi A, Mignouna HD, Zoundjihekpon J, Sangare A, Ahoussou N, Asiedu R. Identification of some Benin republic's Guinea yam (Dioscorea cayenensis/Dioscorea rotundata complex) cultivars using Randomly Amplified Polymorphic DNA. Gen Res Crop Evol 2000 ; 47 : 619-25.

Degras L. L'igname. Plante à tubercule tropicale. Paris: éditions G.-P. Maisonneuve \& Larose, 1986.

Dumont R. La production d'ignames dans un village bariba du Bénin septentrional. Cahiers de la Recherche Développement 1997; (43) : 35-51.

Dumont R, Vernier P. La domestication des ignames (D. cayenensis-D. rotundata) chez l'ethnie bariba du Bénin. Rencontre internationale, gestion des ressources génétiques des plantes en Afrique des savanes, 24-28 février 1997, Bamako (Mali). Paris; Sikasso (Mali); Nogent-sur-Marne (France) : BRG; IER; Solagral, 1997.

Dumont R, Vernier P. Domestication of yams (Dioscorea cayenensis-rotundata) within the Bariba ethnic group in Benin. Outlook on Agriculture $2000 ; 29$ : 137-42.
Dumont R, Dansi A, Vernier P, Zoundjihèkpon J. Biodiversité et domestication des ignames en Afrique de l'Ouest. Pratiques traditionnelles conduisant à Dioscorea rotundata. Collection Repères. Montpellier : Cirad éditions, 2005.

Hamon P. Structure, origine génétique des ignames cultivées du complexe Dioscorea cayenensis-rotundata et domestication des ignames en Afrique de l'Ouest. Thèse sciences naturelles, université Paris XI, Orsay (France), 1987. Travaux et Documents Microédités; 47 Paris : Orstom éditions, 1988.

Hamon P, Brizard JP, Zoundjihekpon J, Duperray $C$, Borgel A. Étude des index d'ADN de huit espèces d'ignames (Dioscorea sp.) par cytométrie en flux. Can J Bot 1992; 70 : 996-1000.

Hildebrand EA. Motives and opportunities for domestication and ethnoarchaeological study in southwest Ethiopa. Journal of Anthropological Archaeology 2003 : 358-75.

Lombard J. Structures de type féodal en Afrique noire, études des dynamismes internes et des relations sociales chez les Bariba du Dahomey. Paris : éditions Mouton, 1965.

Miège J. Contribution à l'étude systématique des Dioscorea $d^{\prime}$ Afrique occidentale. Thèse, faculté des sciences, Paris, 1952.

Miège J. Sur quelques problèmes taxonomiques posés par Dioscorea cayenensis et $D$, rotundata. In: Taxonomic aspects of African Botany Proc. IXth Plenary Meeting of AETFAT, Las Palmas de Gran Canaria, 1978. Las Palmas (Gran Canaria, España) : ed Kunkel, 1979.

Mignouna HD, Mank RA, Ellis THN, et al. A genetic linkage map of Guinea yam (Dioscorea rotundata Poir.) based on AFLP markers. Theor Appl Genet 2002 ; 105 : 716-25.

Mignouna H, Dansi A. 6-Phosphogluconate dehydrogenase (6-PGD) in yam (Dioscorea spp.): variation and classification. Plant Genet Resour News/ 2003a : 27-30.

Mignouna HD, Dansi A, Mayong V. Yam (Diocorea sp.) domestication with the Nago and Fon ethnic group of Benin. Gen Res Crop Evol 2003b ; $50: 519-28$.

Mignouna HD, Abang MM, Wanyera NW, Chikaleke VA, Asiedu R, Thottappilly G. PCR markerbased analysis of wild and cultivated yams (Dioscorea spp.) in Nigeria: genetic relationships for ex situ conservation. Gen Res Crop Evol 2005 ; 52 : 755-63.

Ramser J, Weising K. Lopez- Peralta C, Terhalle W, Terauchi R, Kahl G. Molecular marker-based taxonomy and phylogeny of guinea yam $(D$. rotundata-cayenensis). Genome 1997; 40: 903-15.

Rougerie G. La Côte d'lvoire. Collection Que sais-je? Cinquième édition. Paris: Presses universitaires de France, 1982.

Scarcelli N. Structure et dynamique d'une plante cultivée à multiplication végétative: le cas des ignames au Bénin (Dioscorea sp.). Thèse de doctorat, Biologie des systèmes intégrés, Agronomie-environnement, université Montpellier II, Montpellier, France, 2005. http://www. documentation.ird.fr/intranet/publi/depot/2009-10 13/010035303.pdf
Scarcelli N, Tostain S, Mariac C, et al. Genetic nature of yams (Dioscorea sp.) domesticated by farmers in Benin (West Africa). Gen Res Crop Evol 2006a ; 53 : 121-30.

Scarcelli N, Tostain S, Vigouroux Y, Agbangla C, Daïnou O, Pham JL. Farmer's use of wild relative and sexual reproduction in a vegetatively propaged crop. The case of Benin. Mol Ecol 2006b ; 15: 2421-31.

Stessens J. Analyse technique et économique des systèmes de production agricole au Nord de la Côte d'Ivoire. Doctoraatsproefschrift Nr. 530 aan de Faculteit Landbouwkundige en Toegepaste Biologische Wetenschappen van KULeuven, 2002. www.biw.kuleuven.be/aee/clo/ wp/stessens2002.pdf

Tamiru M, Becker CH, Maass BL. Genetic Diversity in Yam Germplasm from Ethiopia and Their Relatedness to the Main Cultivated Dioscorea Species Assessed by AFLP Markers. 2007. http:// crop.scijournals.org/cgi/content/full/47/4/1744

Terauchi R, Chikaleke VA, Thottappilly G, Hahn SK. Origin and phylogeny of Guinea yams revealed by Rflp analysis of chloroplast DNA and nuclear ribosomal DNA. Theor Appl Genet $1992 ; 83: 743-51$.

Thouvenel JC, Fauquet C. Les viroses de l'igname en Côte d'Ivoire. In: Miège J, Lyonga SN. Yams/lgnames. Compte rendu de la Conférence internationale sur l'igname, International Foundation for Science (IFS), Buéa, Cameroun, 2-6 October 1978. Oxford (UK) : Clarendon Press, 1982.

Tostain S, Agbangla C, Daïnou O. Les ignames Dioscorea abyssinica et $D$. praehensilis en Afrique de l'Ouest. Diversité génétique estimée par des marqueurs Aflp. Annales des Sciences Agronomiques du Bénin $2002 ;(3): 1-20$.

Tostain S, Okry F.K., Baco M.N., et al. « Domestication " des ignames Dioscorea abyssinica dans les sous-préfectures de Sinendé et Banté au Bénin (Afrique de I'Ouest) Annales des Sciences Agronomiques du Bénin 2003 ; (4) : 33-53.

Tostain S, Chaïr H, Scarcelli N. Diversité, origine et dynamique évolutive des ignames cultivées Dioscorea rotundata Poir. au Bénin. Colloque national BRG sur un dialogue pour la diversité génétique, 2004-11-03/2004-11-05, Lyon. Les actes du BRG $2005 ; 5: 465-82$.

Tostain S, Agbangla C, Scarcelli N, et al. Genetic diversity analysis of yam cultivars (Dioscorea rotundata Poir.) in Benin using simple sequence repeat (SSR) markers. Plant Genet Resour 2007 ; $5: 71-81$.

Vernier P, Dossou AR. Adaptation of yam (Dioscorea spp.) cultivation to changing environment and economic constraints in Benin, West Africa. Proceedings of the ISTRC, $12^{\text {th }}$ Symposium on Potential Root Crops for Food and Industrial Resources, Tsukuba (Japan), September 10-16 2000.

Vernier P, Orkwor GC, Dossou AR. Studies on yam domestication and farmers' practices in Benin and Nigeria. Outlook on Agriculture $2003 ; 32: 35-41$.

Vernier P, Dansi A. Participatory assessment of local yam cultivars (D. cayenensis and D. rotundata) in Benin. Plant Genet Resour Newsl 2006: 1-9. 